\title{
Computational Results on Quadratic Functional Model for the Tokens of Nuclear Safety
}

\author{
Joseph Olorunju Omolehin, Lukuman Aminu*, Kamilu Rauf \\ Department of Mathematics, University of Ilorin, Ilorin, Nigeria \\ Email: omolehin_joseph@yahoo.com,,jubril_aminu@yahoo.co.uk,krauf@unilorin.edu.ng
}

Received August 2, 2012; revised October 15, 2012; accepted November 25, 2012

\begin{abstract}
In this work, Nuclear Reactor safety was modeled inform of quadratic functional. The nuclear tokens are structured and used as elements of the control matrix operator in our quadratic functional. The numerical results obtained through Conjugate Gradient Method (CGM) algorithm identify the optimal level of safety required for Nuclear Reactor construction at any particular situation.
\end{abstract}

Keywords: Control Operator; Nuclear Tokens; CGM Algorithm; Optimal

\section{Introduction}

Nuclear reactors accidents occur when the coolant ceases to work, the reactor will be overheated and produced excess heat in form of steam. Most of the internal component of the reactors are made from zirconium in zircalloy cladding used in fuel rods oxidizes in reaction with steam to produce zirconium oxide and hydrogen [1]. When mixed with air, hydrogen is flammable and its detonation will destroy the containment structure which house the reactor. As a result of this, radiation is released to the surrounding causing environmental hazard [2-5]. This work derived the equations for the rate of heat of different reactors from energy balance equations. These equations are structured in parametric form to obtain the basis of the mathematical model solvable by the Conjugate Gradient Method (CGM) algorithm. The obtained numerical results generate the minimal disaster associated with nuclear reactors.

\section{Energy Balance}

The following results were obtained through Energy Balance Equations. See [6]. For the purpose of our study, we shall arrange the equations in terms of the rate of heat.

\subsection{The Energy Balance for Chemical Reactors}

Let us consider arbitrary reactor volume element. From the conservation of energy for a reactor system we obtain the following:

\footnotetext{
"Corresponding author.
}

$$
\begin{aligned}
& \text { (Rate of Energy accumulated }) \\
& =(\text { Rate of Energy of inflow }) \\
& -(\text { Rate of Energy of outflow }) \\
& +(\text { Rate of heat added to the system }) \\
& +(\text { Rate of work done on the system })
\end{aligned}
$$

Equation (1) can be represented mathematically as:

$$
\frac{\mathrm{d} E}{\mathrm{~d} t}=m_{0} \hat{E}_{0}-m_{1} \hat{E}_{1}+\dot{Q}+\dot{W}
$$

where $\hat{E}$ means energy per unit mass, $m_{0}$ is the mass inflow, $m_{1}$ is the mass outflow and $\dot{Q}$ is the rate of heat.

The total rate of work done on a reactor system is expressed as follows:

$$
\dot{W}=\dot{W}_{f}-\dot{W}_{s}+\dot{W}_{b}
$$

where $\dot{W}=$ total rate of work done,

$\dot{W}_{f}=$ rate of work done by flowstream

$\dot{W}_{\mathrm{s}}=$ rate of work done by shaft

$\dot{W}_{b}^{s}=$ rate of work done by boundary

The Rate of work done Flowstream can be represented by $\dot{W}_{f}$ such that

$$
\dot{W}_{f}=v_{0} A_{0} P_{0}-v_{1} A_{1} P_{1}=Q_{0} P_{0}-Q_{0} P_{0}
$$

where $A_{0}=$ area of reactor (inflow), $A_{1}=$ area of reactor (outflow), $v_{0}=$ inflow volume of reactor, $v_{1}=$ outflow volume of reactor, $P_{0}=$ inflow pressure, $P_{1}=$ outflow Pressure, $Q(0)=$ flowrate (in), $Q_{1}=$ flowrate (out), $m=$ mass $\rho_{0}=$ inflow density, $\rho_{1}=$ outflow density and $\rho=$ general density, also 


$$
Q=\frac{m}{\rho}
$$

Substituting (5) in (4) and using the result in (3), we obtain

$$
\dot{W}=m_{0} \frac{P_{0}}{\rho_{0}}-m_{1} \frac{P_{1}}{\rho_{1}}+\dot{W}_{s}+\dot{W}_{b}
$$

The energy terms of total energy composed in Internal $U$, Kinetic $K$ and Potential energy $\phi$ is expressed as:

$$
E=U+K+\phi
$$

Substituting (7) in (2), we obtain

$$
\begin{aligned}
\frac{\mathrm{d}}{\mathrm{d} t}(U+K+\phi)= & m_{0}(\hat{U}+\hat{K}+\hat{\phi})_{0}-m_{1}(\hat{U}+\hat{K}+\hat{\phi})_{1} \\
& +\dot{Q}+\dot{W}_{s}+\dot{W}_{b}
\end{aligned}
$$

but in chemical reactors, only the internal energy is considered with the enthalphy $H=U+P V$ per unit mass, hence 8 becomes

$$
\frac{\mathrm{d}}{\mathrm{d} t} U=m_{0} \hat{H}_{0}-m_{1} \hat{H}_{1}+\dot{Q}+\dot{W}_{s}+\dot{W}_{b}
$$

\subsection{The Batch Reactor}

The batch reactors have no flowstream (i.e. $\left.m_{0} \hat{H}_{0}-m_{1} \hat{H}_{1}=0\right)$. Therefore, Equation (9) in terms of rate of heat becomes

$$
\dot{Q}_{11}=\frac{\mathrm{d} u}{\mathrm{~d} t}-\dot{W}_{s}-\dot{W}_{b}
$$

Neglecting the work done by stirrer because the mixture is not highly viscous, so the stirring operation do not draw significant power, (10) yield

$$
\dot{Q}=\frac{\mathrm{d} u}{\mathrm{~d} t}-\dot{W}_{b}
$$

and we know that $\dot{W}_{b}=-P \frac{\mathrm{d} V_{R}}{\mathrm{~d} t}$, hence (11) becomes

$$
\dot{Q}_{12}=\frac{\mathrm{d} u}{\mathrm{~d} t}+P \frac{\mathrm{d} V_{R}}{\mathrm{~d} t}
$$

For Batch reactor in terms of enthalpy, we have

$$
H=U+P V_{R}
$$

Taking the differential of (13) for $V=V_{R}$ and substituting in (12), we obtain

$$
\dot{Q}_{13}=\frac{\mathrm{d} H}{\mathrm{~d} t}-V_{R} \frac{\mathrm{d} P}{\mathrm{~d} t}
$$

We now consider enthalpy as a function of temperature $T$, pressure $P$ and number of moles $n_{j}$, and express its differentials as

$$
\begin{aligned}
\frac{\mathrm{d} H}{\mathrm{~d} t}= & \left(\frac{\partial H}{\partial T}\right)_{P, n_{j}} \frac{\mathrm{d} T}{\mathrm{~d} t}+\left(\frac{\partial H}{\partial P}\right)_{T, n_{j}} \frac{\mathrm{d} P}{\mathrm{~d} t} \\
& +\sum_{j}\left(\frac{\partial H}{\partial n_{j}}\right)_{T, P, n_{k}} \frac{\mathrm{d} n_{j}}{\mathrm{~d} t}
\end{aligned}
$$

The first partial derivative is the definitions of the heat capacity, $C_{P}$, that is

$$
C_{P}=V_{R} \rho \hat{C}_{P}
$$

The second partial derivative can be expressed as

$$
\left(\frac{\partial H}{\partial P}\right)_{T, n_{j}}=V-T\left(\frac{\partial V}{\partial T}\right)_{P, n_{j}}=V(1-\alpha T)
$$

where $\alpha=\frac{1}{V}\left(\frac{\partial V}{\partial T}\right)_{P, n_{j}}$ is the coefficient of expansion of mixture.

The final partial derivatives are the partial molar enthalpies, $\bar{H}_{j}$

$$
\left(\frac{\partial H}{\partial n_{j}}\right)_{T, P, n_{k}}=\bar{H}_{j}
$$

Substituting (16), (17) and (18) in (15) and using the result in (14), we obtain

$$
\dot{Q}_{14}=V_{R} \rho \hat{C}_{P} \frac{\mathrm{d} T}{\mathrm{~d} t}-\alpha T V_{R} \frac{\mathrm{d} P}{\mathrm{~d} t}+\sum_{j} \bar{H}_{j} \frac{\mathrm{d} n_{j}}{\mathrm{~d} t}
$$

But the material balance for batch reactor is

$$
\frac{\mathrm{d} n_{j}}{\mathrm{~d} t}=R_{j} V_{R}=\sum_{i=1}^{n_{r}} V_{i j} r_{i} V_{R} \quad j=1, \cdots, n_{s}
$$

where $V_{i j}$ is the stoichiometric coefficient for species $j$ and reaction $i, R_{j}$ is the production rate for $j$ th species and $r_{i}$ is the reaction rate for $i$ th reaction.

And the heat of reaction is

$$
\Delta H_{R i}=\sum_{j} V_{i j} \bar{H}_{j}
$$

Substituting (20) and (21) in (19) we obtain

$$
\dot{Q}_{15}=V_{R} \rho \hat{C}_{P} \frac{\mathrm{d} T}{\mathrm{~d} t}-\alpha T V_{R} \frac{\mathrm{d} P}{\mathrm{~d} t}+\sum_{j} \Delta H_{R i} r_{i} V_{R}
$$

The constant-pressure batch reactor is the incompressible-fluid and for $\frac{\mathrm{d} P}{\mathrm{~d} t}=0$ then Equation (22), becomes

$$
\dot{Q}_{16}=V_{R} \rho \hat{C}_{P} \frac{\mathrm{d} T}{\mathrm{~d} t}+\sum_{j} \Delta H_{R i} r_{i} V_{R}
$$

If the heat removal is manipulated to maintain constant reactor temperature, the time derivative in Equation (23) vanishes leaving

$$
\dot{Q}_{21}=\Delta H_{R} r V_{R}
$$


When $C_{A}=$ concentration of species $A, k=$ reaction rate constant, $r=k C_{A}^{2}$ and $H_{R}$ is the enthalpy change on reaction then Equation (24) becomes

$$
\dot{Q}_{22}=\Delta H_{R} k C_{A}^{2} V_{R}
$$

For the constant-volume batch reactor, we considered the pressure as function of temperature, volume and number of moles, and also expressed its differentials as:

$$
\begin{aligned}
\frac{\mathrm{d} P}{\mathrm{~d} t}= & \left(\frac{\partial P}{\partial T}\right)_{V, n_{j}} \frac{\mathrm{d} T}{\mathrm{~d} t}+\left(\frac{\partial P}{\partial V}\right)_{T, n_{j}} \frac{\mathrm{d} V}{\mathrm{~d} t} \\
& +\sum_{j}\left(\frac{\partial P}{\partial n_{j}}\right)_{T, V, n_{k}} \frac{\mathrm{d} n_{j}}{\mathrm{~d} t}
\end{aligned}
$$

For reactor operation at constant volume, $\frac{\mathrm{d} V}{\mathrm{~d} t}=0$, and forming time derivatives, just as we did in (15) to (17) and substituting into Equation (19) gives

$$
\begin{aligned}
\dot{Q}= & {\left[V_{R} \rho \hat{C}_{P}-\alpha T V_{R}\left(\frac{\partial P}{\partial T}\right)_{V, n_{j}}\right] \frac{\mathrm{d} T}{\mathrm{~d} t} } \\
& +\sum_{j}\left[\bar{H}_{j}-\alpha T V_{R}\left(\frac{\partial P}{\partial n_{j}}\right)_{T, V, n_{k}}\right] \frac{\mathrm{d} n_{j}}{\mathrm{~d} t}
\end{aligned}
$$

Note that the first term in brackets is the total constant-volume heat capacity,

$$
\text { that is } C_{v}=V_{R} \rho \hat{C}_{P}-\alpha T V_{R}\left(\frac{\partial P}{\partial T}\right)_{V, n_{j}}
$$

Substitution (28) and the material balance in (20), yields the rate of heat for the energy balance of the Constant-Volume batch Reactor. That is

$$
\begin{aligned}
\dot{Q}_{23}= & V_{R} \rho \hat{C}_{v} \frac{\mathrm{d} T}{\mathrm{~d} t} \\
& +\sum_{j}\left[\Delta H_{R i}-\alpha T V_{R} \sum_{j} V_{i j}\left(\frac{\partial P}{\partial n_{j}}\right)_{T, V, n_{k}}\right] r_{i} V_{R}
\end{aligned}
$$

If we consider a constant volume-ideal gas, where $\alpha T=1$ and $\left(\frac{\partial P}{\partial n_{j}}\right)_{T, V, n_{k}}=\frac{R T}{V}$. Substituting these into (29) gives

$$
\dot{Q}_{24}=V_{R} \rho \hat{C}_{v} \frac{\mathrm{d} T}{\mathrm{~d} t}+\sum_{j}\left[\Delta H_{R i}-R T \bar{V}_{i}\right] r_{i} V_{R}
$$

where

$$
\bar{V}_{i}=\sum_{j} V_{i j}
$$

\subsection{The Continuous Stirred Tank Reactor (CSTR)}

In order to describe the dynamic operation of a CSTR, the energy balance equation must be developed. The CSTR has flowstream, hence using the Equations (8)

$$
\begin{aligned}
\frac{\mathrm{d}}{\mathrm{d} t}(U+K+\phi)= & m_{0}(\hat{U}+\hat{K}+\hat{\rho})_{0}-m_{1}(\hat{U}+\hat{K}+\hat{\phi})_{1} \\
& +\dot{Q}+\dot{W}_{s}+\dot{W}_{b}
\end{aligned}
$$

As in (9) only the internal energy is considered. The out flow stream is flowing out of a well-mixed reactor, thus, the CSTR rate of heat equation using (32) is

$$
\dot{Q}_{25}=\frac{\mathrm{d} u}{\mathrm{~d} t}-Q_{f} \rho_{f} \hat{H}_{f}+Q \rho \hat{H}-\dot{W}_{s}-\dot{W}_{b}
$$

where $Q_{f}=$ volumetric flow rate, $\rho_{f}=$ flow density, $\bar{H}_{f}=$ flow enthalpy, $C_{j f}=$ flow concentration with component $j$ and $Q=$ flow rate.

As before, if sharf work is neglected for the CSTR, Equation (33) becomes

$$
\dot{Q}_{26}=\frac{\mathrm{d} u}{\mathrm{~d} t}-Q_{f} \rho_{f} \hat{H}_{f}+Q \rho \hat{H}+P \frac{\mathrm{d} V_{R}}{\mathrm{~d} t}
$$

and if the enthalpy is considered, we obtain

$$
\dot{Q}_{31}=\frac{\mathrm{d} H}{\mathrm{~d} t}-V_{R} \frac{P}{\mathrm{~d} t}-Q_{f} \rho_{f} \hat{H}_{f}+Q \rho \hat{H}
$$

We consider the change in enthalpy of the continuous stirred tank reactor (CSTR) as a function of temperature, pressure and number of moles, and express its differentials as

$$
\begin{aligned}
\frac{\mathrm{d} H}{\mathrm{~d} t}= & V_{R} \rho \hat{C}_{P} \frac{\mathrm{d} T}{\mathrm{~d} t}+(1-\alpha T) V_{R} \frac{\mathrm{d} P}{\mathrm{~d} t} \\
& +\sum_{j} H_{j} \frac{\mathrm{d} n_{j}}{\mathrm{~d} t}
\end{aligned}
$$

and substituting into Equation (35) gives

$$
\begin{aligned}
\dot{Q}_{32}= & V_{R} \rho \hat{C}_{P} \frac{\mathrm{d} T}{\mathrm{~d} t}-\alpha T V_{R} \frac{\mathrm{d} P}{\mathrm{~d} t}+\sum_{j} \bar{H}_{j} \frac{\mathrm{d} n_{j}}{\mathrm{~d} t} \\
& -Q_{f} \rho_{f} \hat{H}_{f}+Q \rho \hat{H}
\end{aligned}
$$

The material balance for the CSTR is

$$
\frac{\mathrm{d} n_{j}}{\mathrm{~d} t}=Q_{f} C_{j f}-Q C_{j}+\sum_{i} v_{i j} r_{i} V_{R}
$$

After substituting (38) in (37) and re-arrangement yields

$$
\begin{aligned}
\dot{Q}_{33}= & V_{R} \rho \hat{C}_{P} \frac{\mathrm{d} T}{\mathrm{~d} t}-\alpha T V_{R} \frac{\mathrm{d} P}{\mathrm{~d} t}+\sum_{j} \Delta H_{R j} r_{i} V_{R} \\
& -\sum_{j} C_{i f} Q_{f}\left(\bar{H}_{j f}-\bar{H}_{j}\right)
\end{aligned}
$$

The equation of rate of heat for constant-pressure in CSTR that is Incompressible-fluid and its mean in Equation (39) is $\frac{\mathrm{d} p}{\mathrm{~d} t}=0$ and hence we have 


$$
\begin{aligned}
\dot{Q}_{34}= & V_{R} \rho \hat{C}_{P} \frac{\mathrm{d} T}{\mathrm{~d} t}+\sum_{i} \Delta H_{R i} r_{i} V_{R} \\
& -\sum_{j} C_{i f} Q_{f}\left(\bar{H}_{j f}-\bar{H}_{j}\right)
\end{aligned}
$$

From Equation (40), we obtained the equation of rate of heat for constant-volume in CSTR as follows:

$$
\begin{aligned}
\dot{Q}_{35}= & V_{R} \rho \hat{C}_{V} \frac{\mathrm{d} T}{\mathrm{~d} t}+\sum_{i}\left(\Delta H_{R i}-\alpha T V_{R} \sum_{i} v_{i j} P_{n j}\right) r_{i} V_{R} \\
& -\sum_{j} C_{i f} Q_{f}\left(\bar{H}_{j f}-\bar{H}_{j}\right) \\
& -\alpha T V_{R} \sum_{i} P_{n j}\left(C_{i f} Q_{f}-C_{j} Q\right)
\end{aligned}
$$

Also, from Equation (41), the equation of rate of heat for ideal gas is:

$$
\begin{aligned}
\dot{Q}_{36}= & V_{R} \rho \hat{C}_{V} \frac{\mathrm{d} T}{\mathrm{~d} t}+\sum_{i}\left(\Delta H_{R i}-R T \bar{V}_{i}\right) r_{i} V_{R} \\
& -\sum_{j} C_{j f} Q_{f}\left(\bar{H}_{j f}-\bar{H}_{j}\right) \\
& -R T \sum_{j}\left(C_{j f} Q_{f}-C_{j} Q\right)
\end{aligned}
$$

For steady state constant, we have

$$
\hat{C}_{P}, P=P_{f} \text { and } \bar{H}_{j f}-\bar{H}_{j}=\bar{C}_{p s}\left(T_{f}-T\right)
$$

If we re-arrange Equation (39) in the form

$$
\begin{aligned}
& V_{R} \rho \hat{C}_{P} \frac{\mathrm{d} T}{\mathrm{~d} t}-\alpha T V_{R} \frac{\mathrm{d} P}{\mathrm{~d} t} \\
& =\dot{Q}-\sum_{j} \Delta H_{R j} r_{i} V_{R}+\sum_{j} C_{i f} Q_{f}\left(\bar{H}_{j f}-\bar{H}_{j}\right)
\end{aligned}
$$

By setting the Right hand side of (44) equals zero and substituting (43) in the result gives

$$
\dot{Q}_{41}=\sum_{j} \Delta H_{R j} r_{i} V_{R}-\sum_{j} Q_{f} \rho_{f} \hat{C}_{f}\left(T_{f}-T\right)
$$

The heat removal rate of CSTR required bringing CSTR reactor out-flow stream from final temperature $T_{f}$ to temperature $T$ and is given (from 45) by

$$
\dot{Q}_{42}=Q_{f} \rho_{f} \hat{C}_{P} \Delta T
$$

\subsection{The Semi-Batch Reactor}

The development of the semi-batch reactor energy balance follows directly from the CSTR energy balance derivation of the rate of heat by setting $Q=0$. The main results in this paper are therefore summarized below:

Neglecting the Kinetic Energy in Equation (33) of the CSTR, when $Q=0$, we obtain

$$
\dot{Q}_{43}=\frac{\mathrm{d} u}{\mathrm{~d} t}-Q_{f} \rho_{f} \hat{H}_{f}-\dot{W}_{s}-\dot{W}_{b}
$$

Also, by neglecting the Sharf work and consider the Enthalpy when $Q=0$ in (34) and (35) yields

$$
\dot{Q}_{44}=\frac{\mathrm{d} u}{\mathrm{~d} t}+P \frac{\mathrm{d} V_{R}}{\mathrm{~d} t}-Q_{f} \rho_{f} \hat{H}_{f}
$$

and if the enthalpy is used, we obtain

$$
\dot{Q}_{45}=\frac{\mathrm{d} H}{\mathrm{~d} t}-V_{R} \frac{P}{\mathrm{~d} t}-Q_{f} \rho_{f} \hat{H}_{f}
$$

By setting $Q=0$ in Equations (37) and (39) respectively, we have the enthalpy change of semi-batch reactor as

$$
\begin{aligned}
\dot{Q}_{46}= & V_{R} \rho \hat{C}_{P} \frac{\mathrm{d} T}{\mathrm{~d} t}-\alpha T V_{R} \frac{\mathrm{d} P}{\mathrm{~d} t} \\
& +\sum_{j} \bar{H}_{j} \frac{\mathrm{d} n_{j}}{\mathrm{~d} t}-Q_{f} \rho_{f} \bar{H}_{f}
\end{aligned}
$$

and

$$
\begin{aligned}
\dot{Q}_{51}= & V_{R} \rho \hat{C}_{P} \frac{\mathrm{d} T}{\mathrm{~d} t}-\alpha T V_{R} \frac{\mathrm{d} P}{\mathrm{~d} t}+\sum_{i} \Delta H_{R i} r_{i} V_{R} \\
& -\sum_{j} C_{j f} Q_{f}\left(\bar{H}_{j f}-\bar{H}_{j}\right)
\end{aligned}
$$

The constant pressure semi-batch reactor is the incompressible-fluid batch reactor and in Equation (51) when $\frac{\mathrm{d} P}{\mathrm{~d} t}=0$, we obtain

$$
\begin{aligned}
\dot{Q}_{52}= & V_{R} \rho \hat{C}_{P} \frac{\mathrm{d} T}{\mathrm{~d} t}+\sum_{i} \Delta H_{R i} r_{i} V_{R} \\
& -\sum_{j} C_{j f} Q_{f}\left(\bar{H}_{j f}-\bar{H}_{f}\right)
\end{aligned}
$$

For steady state semi-batch reactor when $\hat{C}_{P}$ is constant, we have

$$
\dot{Q}_{53}=V_{R} \rho \hat{C}_{P} \frac{\mathrm{d} T}{\mathrm{~d} t}+\sum_{i} \Delta H_{R i} r_{i} V_{R}-Q_{f} \rho_{f} \hat{C}_{P}\left(T_{f}-T\right)
$$

The equation is derived from the energy balance equation for Plug-flow reactor (PFR) single phase for rate of heat, and is given by:

$$
\dot{Q}_{54}=Q \rho \hat{C}_{P} \frac{\mathrm{d} T}{\mathrm{~d} V}+Q(1-\alpha T) \frac{\mathrm{d} P}{\mathrm{~d} V}+\sum_{i} \Delta H_{R i} r_{i}
$$

Neglecting pressure drop or ideal gas for PFR and from (54), for an Ideal gas $\alpha T=1$ we have

$$
\dot{Q}_{55}=Q \rho \hat{C}_{P} \frac{\mathrm{d} T}{\mathrm{~d} V}+\sum_{i} \Delta H_{R i} r_{i}
$$

The rate of heat equation of PFR for Incompressible fluid is obtain by setting $\alpha T \frac{\mathrm{d} P}{\mathrm{~d} V}=0$ in Equation (54)

$$
\dot{Q}_{56}=Q \rho \hat{C}_{P} \frac{\mathrm{d} T}{\mathrm{~d} V}+Q \frac{\mathrm{d} P}{\mathrm{~d} V}+\sum_{i} \Delta H_{R i} r_{i}
$$

The remaining six existing equations related to the rate of heat of a reactor for temperature of heat transfer medium $T_{a}$ are as stated below:

$$
\dot{Q}_{61}=-\frac{k(T)}{1+k(T) \tau} C_{A f} \Delta H_{R}
$$




$$
\begin{gathered}
\dot{Q}_{62}=\frac{C_{P S}}{\tau}\left(T-T_{f}\right) \\
\dot{Q}_{63}=\frac{2}{R} \cup^{O}\left(T_{a}-T\right) \\
\dot{Q}_{64}=\bigcup^{O} 2 \pi R \Delta_{Z}\left(T_{a}-T\right) \\
\dot{Q}_{65}=K_{1}\left[C_{A}-\frac{1}{k_{1}}\left(C_{A f}-C_{A}\right)\right] \Delta H_{R} V_{R}-Q_{f} \rho \hat{C}_{P}\left(T_{f}-T\right) \\
\dot{Q}_{66}=\frac{\mathrm{d}}{\mathrm{d} V}(Q \rho \hat{H})
\end{gathered}
$$

The equations derived above from the energy balance equation of chemical reactors [7] are thirty; namely: (10), (12), (14), (19), (22)-(25), (29), (30), (33)-(35), (37), (39)-(42) and (45)-(56). These equations with the six existing equations, namely (57)-(62), were structured into mathematical model in form of quadratic functional. The model with some given existing nuclear tokens were solved by the Conjugate Gradient Method algorithm, with MATLAB as a support soft-ware.

\section{The Gradient Method (CGM) Algorithm}

The CGM algorithm was originally developed by Hestenes and Stiefel [8] to minimize and solve problems in quadratic functional of the form:

$$
f(X)=f_{0}+\langle a, X\rangle_{H}+\frac{1}{2}\langle X, A X\rangle_{H}
$$

where $f_{0}$, is a constant in $H, x$ is a vector in $H . A$ is a positive definite, symmetric and constant matrix operator.

It has a well worked out theory with an elegant convergence profile. No approximation is used in the proving its convergency.

\subsection{Property of Conjugate Gradient Method (CGM) Algorithm}

Some of the several properties of CGM are:

1) It has a quadratic convergence property that is for a quadratic functional on an $n$-dimensional Hilbert space, it converges in at most $n$ steps.

2) It requires a relatively small increase in computer time per iteration and memory space.

3) It has a well worked out theory.

\subsection{Algorithm}

The first element of the descent sequence $x_{0}$ is simply guessed. The remaining members of the sequence are then found as follows:

$$
P_{0}=-g_{0}=-\left(a+A_{0}\right)
$$

$$
\begin{gathered}
X_{i+1}=X_{i}+\alpha_{i} P_{i} \quad \alpha_{i}=\frac{\left\langle g_{i}, g_{i}\right\rangle}{\left\langle P_{i}, A P_{i}\right\rangle} \\
g_{i+1}=g_{i}+\alpha_{i} P_{i} \\
P_{i+1}=-g_{i+1}+\beta_{i} P_{i} \quad \beta_{i}=\frac{\left\langle g_{i+1}, g_{i+1}\right\rangle}{\left\langle g_{i}, g_{i}\right\rangle}
\end{gathered}
$$

where $g_{i}$ is the gradient at the ith element of the descent sequence $X_{i}$.

It has been proved that the algorithm converges at most, in $n$ iteration in a well posed problem and the convergence rate is given as:

$$
E\left(x_{n}\right)=\left\{\frac{1-\frac{m}{M}}{1+\frac{m}{M}}\right\}^{2 n} E\left(x_{0}\right)
$$

where $m$ and $M$ are smallest and spectrums of matrix $A$ respectively. That is, for an $\mathrm{n}$ dimensional problem, the algorithm will converge in at most $n$ iterations [9].

\section{Computational Results}

Our model is:

$$
f(X)=f_{0}+\langle a, X\rangle_{H}+\frac{1}{2}\langle X, A X\rangle_{H}
$$

where $X \in \mathbb{R}^{6}$ i.e., $X=\left(\begin{array}{llllll}x_{1} & x_{2} & x_{3} & x_{4} & x_{5} & x_{6}\end{array}\right)^{\mathrm{T}}$, $a=\left(\begin{array}{llllll}1 & 1 & 1 & 1 & 1 & 1\end{array}\right)^{\mathrm{T}}, \quad f_{0}=1$ and

$$
\boldsymbol{A}=\left(\begin{array}{llllll}
a_{11} & a_{12} & a_{13} & a_{14} & a_{15} & a_{16} \\
a_{21} & a_{22} & a_{23} & a_{24} & a_{25} & a_{26} \\
a_{31} & a_{32} & a_{33} & a_{34} & a_{35} & a_{36} \\
a_{41} & a_{42} & a_{43} & a_{44} & a_{45} & a_{46} \\
a_{51} & a_{52} & a_{53} & a_{54} & a_{55} & a_{56} \\
a_{61} & a_{62} & a_{63} & a_{64} & a_{65} & a_{66}
\end{array}\right)
$$

where $a_{i j}=$ values of the rate of heat, $i=1,2,3,4,5,6$; and $j=1,2,3,4,5,6$.

Numerical values are now calculated for our parameters or tokens. In all cases our initial guess is 0 vector that is $x_{0}=\left(\begin{array}{llllll}0 & 0 & 0 & 0 & 0 & 0\end{array}\right)^{\mathrm{T}}$ and the results are as shown below:

Problem 1 (For arbitrary tokens)

$$
\boldsymbol{A}=\left(\begin{array}{cccccc}
360 & 2 & 0 & 173 & 173 & 173 \\
10 & 208 & 32 & 37 & 34 & -2 \\
3 & 173 & 173 & 16 & 9 & -3 \\
2 & 0 & 173 & 173 & 173 & 173 \\
0 & 1 & 0 & -2 & 113 & -2 \\
-10 & 0 & 1 & 1 & 5 & 108
\end{array}\right)
$$

Problem 2 (For arbitrary tokens) 


$$
\boldsymbol{A}=\left(\begin{array}{cccccc}
108.422 & 2.0 & 0.4032 & 173.16 & 173.16 & 173.16 \\
10.316 & 113.1035 & 31.5015 & 36.5015 & 34.4964 & -1.7986 \\
3 . & 173.151 & 173.169 & 15.813 & 9 . & -2.9065 \\
2.0935 & 0.4967 & 173.151 & 173.2535 & 173.151 & 173.151 \\
0.0003 & 0.6565 & -0.1720 & -1.7986 & 207.6612 & -1.7986 \\
-10.4951 & 0.0201 & 0.8280 & 0.8280 & 5.3300 & 359.991
\end{array}\right)
$$

\section{Problem 3 (For nuclear tokens)}

$$
\boldsymbol{A}=\left(\begin{array}{cccccc}
59.9559 & 4.0000 & -24.5726 & 4.0935 & 0.8280 & -0.3726 \\
4.0000 & 52.9559 & -24.5816 & -24.5636 & -31.7636 & -24.5726 \\
-24.5726 & -24.5816 & 38.5015 & -1.0887 & 3.0935 & 3.5910 \\
4.0935 & -24.5636 & -1.0887 & 38.5015 & 9.0000 & -0.3726 \\
0.8280 & -31.7636 & 3.0935 & 9.0000 & 37.5015 & 0.0845 \\
-0.3726 & -24.5726 & 3.5910 & -0.3726 & 0.0845 & 9.9289
\end{array}\right)
$$

\section{Problem 4 (For nuclear tokens)}

$$
\boldsymbol{A}=\left(\begin{array}{cccccc}
80.4469 & 4.0000 & -24.5726 & 4.0935 & 0.8280 & -0.3726 \\
4.0000 & 59.9559 & -24.5816 & -24.5636 & -31.7636 & -24.5726 \\
-24.5726 & -24.5816 & 52.9559 & -1.0887 & 3.0935 & 3.5910 \\
4.0935 & -24.5636 & -1.0887 & 38.5015 & 9.0000 & -0.3726 \\
0.8280 & -31.7636 & 3.0935 & 9.0000 & 38.5015 & 9.9289 \\
-0.3726 & -24.5726 & 3.5910 & -0.3726 & 9.9289 & 37.5015
\end{array}\right)
$$

\subsection{Tables of Results}

The following tables are Table 1 for Problem 1, Table 2 for problem 2, Table 3 for problem 3 and Table 4 for problem 4 respectively.

\subsection{Discussion of Results}

The initial nuclear tokens used in problems 1 and 2 to represent the vectors and control operators of the quadratic model were arbitrary. Our results clearly shown that arbitrary composition of nuclear tokens will not guarantee safety. This is evidence from Tables 1 and $\mathbf{2}$ (non convergence) which did not satisfied the properties of the CGM algorithm. See [10].

After restructuring, nuclear tokens were used as the vectors and control operators of the quadratic model to generate problems 3 and 4 and were solved using the CGM algorithm. We were able to get two results that converge (Tables 3 and $\mathbf{4}$ ). The convergency satisfied the properties of the CGM algorithm, which shows good results. See [11] and [12].

\section{Conclusions}

The nuclear reactors tokens are the main components that make up the reactors, example of the components are Internal energy, Reactor volume, Molar mass and so on,

\begin{tabular}{|c|c|c|c|}
\hline $\begin{array}{l}\text { Number of } \\
\text { Iteration }\end{array}$ & $\begin{array}{c}\text { Minimizing } \\
\text { Vectors } X\end{array}$ & $\begin{array}{l}\text { Objective } \\
\text { Function }\end{array}$ & Gradient \\
\hline 1 & $x_{1}$ to $x_{6}=$ nill & nill & 2.449 \\
\hline 2 & $x_{1}$ to $x_{6}=$ nill & $0: 99$ & 1.704 \\
\hline 3 & $x_{1}$ to $x_{6}=$ nill & $0: 99$ & 5.016 \\
\hline 4 & $x_{1}$ to $x_{6}=$ nill & $1: 01$ & 9.724 \\
\hline 5 & $x_{1}$ to $x_{6}=$ nill & $1: 06$ & 14.045 \\
\hline 6 & $x_{1}$ to $x_{6}=$ nill & $1: 13$ & 17.715 \\
\hline 7 & $x_{1}$ to $x_{6}=$ nill & $1: 22$ & 20.808 \\
\hline 8 & $x_{1}$ to $x_{6}=$ nill & $1: 33$ & 23.440 \\
\hline 9 & $x_{1}$ to $x_{6}=$ nill & $1: 45$ & 25.713 \\
\hline 10 & $x_{1}$ to $x_{6}=$ nill & $1: 58$ & 27.704 \\
\hline 11 & $x_{1}$ to $x_{6}=$ nill & $1: 73$ & 29.474 \\
\hline 12 & $x_{1}$ to $x_{6}=$ nill & $1: 89$ & 31.067 \\
\hline 13 & $x_{1}$ to $x_{6}=$ nill & $2: 06$ & 32.519 \\
\hline 14 & $x_{1}$ to $x_{6}=$ nill & $2: 23$ & 33.855 \\
\hline 15 & $x_{1}$ to $x_{6}=$ nill & $2: 42$ & 35.097 \\
\hline 16 & $x_{1}$ to $x_{6}=$ nill & $2: 61$ & 36.260 \\
\hline 17 & $x_{1}$ to $x_{6}=$ nill & $2: 81$ & 37.358 \\
\hline
\end{tabular}
which produces the rate of heat of the reactor that causes
Table 1. Generated from problem 1. 


\section{Continued}

\begin{tabular}{|c|c|c|c|}
\hline 18 & $x_{1}$ to $x_{6}=$ nill & $3: 02$ & 38.401 \\
\hline 19 & $x_{1}$ to $x_{6}=$ nill & $3: 24$ & 39.397 \\
\hline 20 & $x_{1}$ to $x_{6}=$ nill & $3: 46$ & 40.353 \\
\hline 21 & $x_{1}$ to $x_{6}=$ nill & $3: 68$ & 41.275 \\
\hline 22 & $x_{1}$ to $x_{6}=$ nill & $3: 91$ & 42.167 \\
\hline 23 & $x_{1}$ to $x_{6}=$ nill & $4: 15$ & 43.032 \\
\hline 24 & $x_{1}$ to $x_{6}=$ nill & $4: 39$ & 43.873 \\
\hline 25 & $x_{1}$ to $x_{6}=$ nill & $4: 64$ & 44.694 \\
\hline 26 & $x_{1}$ to $x_{6}=$ nill & $4: 89$ & 45.495 \\
\hline 27 & $x_{1}$ to $x_{6}=$ nill & $5: 15$ & 46.279 \\
\hline 28 & $x_{1}$ to $x_{6}=$ nill & $5: 41$ & 47.046 \\
\hline 29 & $x_{1}$ to $x_{6}=$ nill & $5: 68$ & 47.799 \\
\hline 30 & $x_{1}$ to $x_{6}=$ nill & $5: 95$ & 48.537 \\
\hline 31 & $x_{1}$ to $x_{6}=$ nill & $6: 22$ & 49.261 \\
\hline 32 & $x_{1}$ to $x_{6}=$ nill & $6: 50$ & 49.973 \\
\hline 33 & $x_{1}$ to $x_{6}=$ nill & $6: 78$ & 50.671 \\
\hline 34 & $x_{1}$ to $x_{6}=$ nill & $7: 06$ & 51.358 \\
\hline 35 & $x_{1}$ to $x_{6}=$ nill & $7: 35$ & 52.033 \\
\hline 36 & $x_{1}$ to $x_{6}=$ nill & $7: 64$ & 52.696 \\
\hline 37 & $x_{1}$ to $x_{6}=$ nill & $7: 93$ & 53.347 \\
\hline 38 & $x_{1}$ to $x_{6}=$ nill & $8: 23$ & 53.988 \\
\hline 39 & $x_{1}$ to $x_{6}=$ nill & $8: 52$ & 54.617 \\
\hline 40 & $x_{1}$ to $x_{6}=$ nill & $8: 82$ & 55.235 \\
\hline 41 & $x_{1}$ to $x_{6}=$ nill & $9: 13$ & 55.842 \\
\hline 42 & $x_{1}$ to $x_{6}=$ nill & $9: 43$ & 56.439 \\
\hline 43 & $x_{1}$ to $x_{6}=$ nill & $9: 74$ & 57.024 \\
\hline 44 & $x_{1}$ to $x_{6}=$ nill & $10: 04$ & 57.599 \\
\hline 45 & $x_{1}$ to $x_{6}=$ nill & $10: 35$ & 58.164 \\
\hline 46 & $x_{1}$ to $x_{6}=$ nill & $10: 66$ & 58.718 \\
\hline 47 & $x_{1}$ to $x_{6}=$ nill & $10: 97$ & 59.263 \\
\hline 48 & $x_{1}$ to $x_{6}=$ nill & $11: 28$ & 59.797 \\
\hline 49 & $x_{1}$ to $x_{6}=$ nill & $11: 60$ & 60.321 \\
\hline 50 & $x_{1}$ to $x_{6}=$ nill & $11: 91$ & 60.836 \\
\hline \multirow{6}{*}{51} & $x_{1}=0.0737$ & \multirow{6}{*}{ nill } & \multirow{6}{*}{ nill } \\
\hline & $x_{2}=-0.0563$ & & \\
\hline & $x_{3}=0.0192$ & & \\
\hline & $x_{4}=0.4714$ & & \\
\hline & $x_{5}=-0.1788$ & & \\
\hline & $x_{6}=-0.1705$ & & \\
\hline
\end{tabular}

Table 2. Generated from problem 2.

\begin{tabular}{|c|c|c|c|}
\hline $\begin{array}{l}\text { Number of } \\
\text { Iteration }\end{array}$ & $\begin{array}{c}\text { Minimizing } \\
\text { Vectors } X\end{array}$ & $\begin{array}{l}\text { Objective } \\
\text { Function }\end{array}$ & Gradient \\
\hline 1 & $x_{1}$ to $x_{6}=$ nill & nill & 2.449 \\
\hline 2 & $x_{1}$ to $x_{6}=$ nill & 0:99 & 1.110 \\
\hline 3 & $x_{1}$ to $x_{6}=$ nill & $0: 99$ & 2.598 \\
\hline 4 & $x_{1}$ to $x_{6}=$ nill & $1: 00$ & 3.647 \\
\hline 5 & $x_{1}$ to $x_{6}=$ nill & $1: 01$ & 3.931 \\
\hline 6 & $x_{1}$ to $x_{6}=$ nill & $1: 02$ & 3.987 \\
\hline 7 & $x_{1}$ to $x_{6}=$ nill & $1: 04$ & 4.060 \\
\hline 8 & $x_{1}$ to $x_{6}=$ nill & $1: 05$ & 4.240 \\
\hline 9 & $x_{1}$ to $x_{6}=$ nill & $1: 06$ & 4.564 \\
\hline 10 & $x_{1}$ to $x_{6}=$ nill & $1: 07$ & 5.050 \\
\hline 11 & $x_{1}$ to $x_{6}=$ nill & $1: 09$ & 5.705 \\
\hline 12 & $x_{1}$ to $x_{6}=$ nill & $1: 10$ & 6.538 \\
\hline 13 & $x_{1}$ to $x_{6}=$ nill & $1: 12$ & 7.551 \\
\hline 14 & $x_{1}$ to $x_{6}=$ nill & $1: 14$ & 8.746 \\
\hline 15 & $x_{1}$ to $x_{6}=$ nill & $1: 17$ & 10.120 \\
\hline 16 & $x_{1}$ to $x_{6}=$ nill & $1: 20$ & 11.665 \\
\hline 17 & $x_{1}$ to $x_{6}=$ nill & $1: 23$ & 13.375 \\
\hline 18 & $x_{1}$ to $x_{6}=$ nill & $1: 27$ & 15.229 \\
\hline 19 & $x_{1}$ to $x_{6}=$ nill & $1: 31$ & 17.217 \\
\hline 20 & $x_{1}$ to $x_{6}=$ nill & $1: 36$ & 19.319 \\
\hline 21 & $x_{1}$ to $x_{6}=$ nill & $1: 41$ & 21.516 \\
\hline 22 & $x_{1}$ to $x_{6}=$ nill & $1: 47$ & 23.788 \\
\hline 23 & $x_{1}$ to $x_{6}=$ nill & $1: 54$ & 26.117 \\
\hline 24 & $x_{1}$ to $x_{6}=$ nill & $1: 61$ & 28.485 \\
\hline 25 & $x_{1}$ to $x_{6}=$ nill & $1: 69$ & 30.874 \\
\hline 26 & $x_{1}$ to $x_{6}=$ nill & $1: 78$ & 33.272 \\
\hline 27 & $x_{1}$ to $x_{6}=$ nill & $1: 87$ & 35.663 \\
\hline 28 & $x_{1}$ to $x_{6}=$ nill & $1: 96$ & 38.038 \\
\hline 29 & $x_{1}$ to $x_{6}=$ nill & $2: 07$ & 40.387 \\
\hline 30 & $x_{1}$ to $x_{6}=$ nill & $2: 17$ & 42.701 \\
\hline 31 & $x_{1}$ to $x_{6}=$ nill & $2: 29$ & 44.974 \\
\hline 32 & $x_{1}$ to $x_{6}=$ nill & $2: 41$ & 47.201 \\
\hline
\end{tabular}




\section{Continued}

\begin{tabular}{|c|c|c|c|}
\hline 33 & $x_{1}$ to $x_{6}=$ nill & $2: 53$ & 49.377 \\
\hline 34 & $x_{1}$ to $x_{6}=$ nill & $2: 66$ & 51.501 \\
\hline 35 & $x_{1}$ to $x_{6}=$ nill & $2: 80$ & 53.569 \\
\hline 36 & $x_{1}$ to $x_{6}=$ nill & $2: 94$ & 55.579 \\
\hline 37 & $x_{1}$ to $x_{6}=$ nill & 3:09 & 57.532 \\
\hline 38 & $x_{1}$ to $x_{6}=$ nill & $3: 24$ & 59.427 \\
\hline 39 & $x_{1}$ to $x_{6}=$ nill & $3: 39$ & 61.263 \\
\hline 40 & $x_{1}$ to $x_{6}=$ nill & $3: 55$ & 63.042 \\
\hline 41 & $x_{1}$ to $x_{6}=$ nill & $3: 71$ & 64.764 \\
\hline 42 & $x_{1}$ to $x_{6}=$ nill & $3: 88$ & 66.436 \\
\hline 43 & $x_{1}$ to $x_{6}=$ nill & 4:05 & 68.041 \\
\hline 44 & $x_{1}$ to $x_{6}=$ nill & $4: 23$ & 69.599 \\
\hline 45 & $x_{1}$ to $x_{6}=$ nill & $4: 41$ & 71.104 \\
\hline 46 & $x_{1}$ to $x_{6}=$ nill & $4: 59$ & 72.559 \\
\hline 47 & $x_{1}$ to $x_{6}=$ nill & $4: 78$ & 73.965 \\
\hline 48 & $x_{1}$ to $x_{6}=$ nill & 4:97 & 75.324 \\
\hline 49 & $x_{1}$ to $x_{6}=$ nill & $5: 16$ & 76.636 \\
\hline 50 & $x_{1}$ to $x_{6}=$ nill & $5: 36$ & 77.904 \\
\hline \multirow{6}{*}{51} & $x_{1}=-0.2289$ & \multirow{6}{*}{$5: 56$} & \multirow{6}{*}{ nill } \\
\hline & $x_{2}=-0.0724$ & & \\
\hline & $x_{3}=0.2464$ & & \\
\hline & $x_{4}=-0.0587$ & & \\
\hline & $x_{5}=0.1224$ & & \\
\hline & $x_{6}=0.0549$ & & \\
\hline
\end{tabular}

Table 3. Generated from problem 3.

\begin{tabular}{cccc}
$\begin{array}{c}\text { Number of } \\
\text { Iteration }\end{array}$ & $\begin{array}{c}\text { Minimizing } \\
\text { Vectors } X\end{array}$ & $\begin{array}{c}\text { Objective } \\
\text { Function }\end{array}$ & Gradient \\
\hline & $x_{1}=0.000$ & & \\
$x_{2}=0.000$ & & \\
1 & $x_{3}=0.000$ & nill & \\
& $x_{4}=0.000$ & & \\
& $x_{5}=0.000$ & & \\
& $x_{6}=0.000$ & \\
\hline
\end{tabular}

\section{Continued}

\begin{tabular}{|c|c|c|c|}
\hline \multirow{6}{*}{2} & $x_{1}=-0.261$ & \multirow{6}{*}{0.22} & \multirow{6}{*}{19.149} \\
\hline & $x_{2}=-0.261$ & & \\
\hline & $x_{3}=-0.261$ & & \\
\hline & $x_{4}=-0.261$ & & \\
\hline & $x_{5}=-0.261$ & & \\
\hline & $x_{6}=-0.261$ & & \\
\hline \multirow{6}{*}{3} & $x_{1}=0.033$ & \multirow{6}{*}{1.28} & \\
\hline & $x_{2}=0.174$ & & \\
\hline & $x_{3}=0.108$ & & \\
\hline & $v-0061$ & & \\
\hline & $x_{5}=0.071$ & & \\
\hline & $x_{6}=0.118$ & & \\
\hline \multirow{6}{*}{4} & $x_{1}=-0.013$ & \multirow{6}{*}{1.21} & \multirow{6}{*}{1.978} \\
\hline & $x_{2}=0.124$ & & \\
\hline & $x_{3}=0.070$ & & \\
\hline & $x_{4}=0.045$ & & \\
\hline & $x_{5}=0.068$ & & \\
\hline & $x_{6}=0.127$ & & \\
\hline \multirow{6}{*}{5} & $x_{1}=-0.024$ & \multirow{6}{*}{1.16} & \multirow{6}{*}{0.234} \\
\hline & $x_{2}=0.106$ & & \\
\hline & $x_{3}=0.007$ & & \\
\hline & $x_{4}=0.032$ & & \\
\hline & $x_{5}=0.060$ & & \\
\hline & $x_{6}=0.147$ & & \\
\hline \multirow{6}{*}{6} & $x_{1}=-0.022$ & \multirow{6}{*}{1.16} & \multirow{6}{*}{0.006} \\
\hline & $x_{2}=0.103$ & & \\
\hline & $x_{3}=0.008$ & & \\
\hline & $x_{4}=0.031$ & & \\
\hline & $x_{5}=0.053$ & & \\
\hline & $x_{6}=0.1152$ & & \\
\hline \multirow{6}{*}{7} & $x_{1}=-0.022$ & \multirow{6}{*}{1.16} & \multirow{6}{*}{0.000} \\
\hline & $x_{2}=0.103$ & & \\
\hline & $x_{3}=0.008$ & & \\
\hline & $x_{4}=0.032$ & & \\
\hline & $x_{5}=0.053$ & & \\
\hline & $x_{6}=0.152$ & & \\
\hline
\end{tabular}


Table 4. Generated from problem 4.

\begin{tabular}{|c|c|c|c|}
\hline $\begin{array}{l}\text { Number of } \\
\text { Iteration }\end{array}$ & $\begin{array}{c}\text { Minimizing } \\
\text { Vectors } X\end{array}$ & $\begin{array}{l}\text { Objective } \\
\text { Function }\end{array}$ & Gradient \\
\hline 1 & $\begin{array}{l}x_{1}=0.000 \\
x_{2}=0.000 \\
x_{3}=0.000 \\
x_{4}=0.000 \\
x_{5}=0.000 \\
x_{6}=0.000\end{array}$ & nill & 2.449 \\
\hline 2 & $\begin{array}{l}x_{1}=-0.053 \\
x_{2}=-0.053 \\
x_{3}=-0.053 \\
x_{4}=-0.053 \\
x_{5}=-0.053 \\
x_{6}=-0.053\end{array}$ & 0.84 & 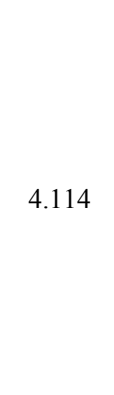 \\
\hline 3 & $\begin{array}{l}x_{1}=-0.071 \\
x_{2}=-0.321 \\
x_{3}=-0.201 \\
x_{4}=-0.163 \\
x_{5}=-0.153 \\
x_{6}=-0.162\end{array}$ & 0.46 & 2.970 \\
\hline 4 & $\begin{array}{l}x_{1}=-0.040 \\
x_{2}=-0.367 \\
x_{3}=-0.217 \\
x_{4}=-0.211 \\
x_{5}=-0.206 \\
x_{6}=-0.199\end{array}$ & 0.38 & 1.930 \\
\hline 5 & $\begin{array}{l}x_{1}=-0.040 \\
x_{2}=-0.395 \\
x_{3}=-0.198 \\
x_{4}=-0.230 \\
x_{5}=-0.226 \\
x_{6}=-0.209\end{array}$ & 0.35 & 0.092 \\
\hline
\end{tabular}

Continued

$\begin{array}{ll}x_{1}=-0.040 & \\ x_{2}=-0.395 & \\ x_{3}=-0.198 & 0.35 \\ x_{4}=-0.228 & 0.019 \\ x_{5}=-0.228 & \\ x_{6}=-0.209 & \\ x_{1}=-0.040 & \\ x_{2}=-0.395 & \\ x_{3}=-0.198 \\ x_{4}=-0.228 \\ x_{5}=-0.228 \\ x_{6}=-0.209\end{array}$

the accidents. See [13-20].

Our results clearly indicate that if the nuclear tokens are used for the structured model, which are used for the construction of nuclear reactors, then the nuclear safety will be maximized while the disaster will be minimized.

\section{REFERENCES}

[1] Nuclear Energy Agency, "Nuclear Fuel Behaviour in Loss-of-Coolant-Accident (LOCA) Conditions," 2009, p. 141.

http://wwwoecd-nea.org/nsd/reports/2009/nea6846-LOC A.pdf

[2] A. C. Kadak, et al., "A Response to the Environmental and Economic Challenge of Global Warming," Handbook, Massachusetts Institute of Technology, 1998, pp. 1-6.

[3] Encyclopaedia Britannica, "Windmill Power," Ultimate Refrence Suite: Chicago Encyclopaedia Britannica, Knoxville, 2010.

[4] Encyclopaedia Britannica, "Hydroelectric Power," Ultimate Refrence Suite: Chicago Encyclopaedia Britannica, Knoxville, 2010.

[5] C. K. Otto, "Fossil Fuel Power," University of Tennessee, Ultimate Refrence Suite: Chicago Encyclopaedia Britannica, Knoxville, 2011.

[6] "The Enargy Balance for Chemical Reactors," 1st Edition, Nob Hill Publishing LLC, Madison, 2011, pp. 1-182. www.che.wisc.edu/home/jbraw/chemreacfun/chb/slides-e nbal.pdf

[7] J. O. Omolehin, L. Aminu and K. Rauf, "Control Approach to Nuclear Safety," Applied Mathematics-Open Special Issues "Optimization”, Scientific Research, 2012. http://www.scirp.org/journal/am

[8] J. O. Omolehin, K. Rauf and D. J. Evans, "Conjugate 
Gradient Method Approach to Queue Theory," International Journal of Computer Mathematics, Vol. 82, No. 7, 2005, pp. 829-835. http://www.tandf.co.uk/journals

[9] J. O. Omolehin, "On the Control of Reaction Diffusion Equation," Ph.D. Thesis, University of Ilorin, Ilorin, 1991.

[10] T. Gland, "Constrained Optimization Using Multiplier Methods with Application to Control Problems," University of Lund, Lund, Report No. 7603, 1976.

[11] International Atomic Energy Agency, "Structure of Nuclear Power Plant Design Characteristics in the IAEA Power Reactor Information System," IAEA, Vienna, 2007, pp. 9-17.

[12] S. Frank, "Nuclear Reactors," Kennesaw State University, Kennesaw, 2009, pp. 1-13. www.chemcases.com/nuclear/nc-10.html

[13] Wikipedia, "Boiling Water Reactor Safety System," The Free Encyclopedia, 2011, pp. 2-9. en.wikipedia.org/wiki/Boiling-water-reactor-safety-syste $\mathrm{ms}$

[14] S. Ashok, "Solar Energy," Department of Engineering, Pennsylvania State University, Ultimate Refrence Suite: Chicago Encyclopaedia Britannica, Knoxville, 2011.
[15] J. S. Walker, "The Three Mile Island: A Nuclear Crises in Historical Perspective," University of California Press, Berkeley, 2004, pp. 216-265.

[16] “The Chernobyl Facts," 2011. http://www.chernobyl-interntional.org/documents/cherno bylfacts2.pdf

[17] Wikipedia, "Fukushima Daiichi Nuclear Reactor Disaster," The Free Encyclopedia, 2011, pp. 5-27. en.wikipedia.org/wiki/Fukushima-Daiichi-nuclear-diaster

[18] Wikipedia, "Energy in Japan," The Free Encyclopedia, 2009 , p. 1 en.wikipedia.org/wiki/Electricity-sector-in-Japan

[19] International Atomic Energy Agency, "Mitigation of Hydrogen Hazard in Water Cooled Power Reactors," IAEA, Vienna, 2001, pp. 3-10.

http://www-pub.iaea.org/MTCD/publicatuion/PDF/te-119 6-prn.pdf

[20] G. H. Brian, "Energy Equations for Reactors," Department of Chemical Engineering and Material Science, University of California, Lecture Note, Davis, 2010, pp. 9-13. 